\title{
14
}

\section{Organisational Culture and Modes of Conflict Behaviour}

S. Ayestarán, C. Martínez-Taboada and J. Arróspide

Department of Social Psychology

University of the Basque Country

Apartado 1249

20080 San Sebastián, Spain

Tel.: 3443310600 Fax: 3443311055

e-mail: pspayets@ss.ehu.es

\section{Garcia}

Department of Social Psychology

London School of Economics

Houghton Street, London WC2A 2AE, UK.

Tel. 44171955 7695/7767 Fax: 441719557565

e-mail:l.garcia@Lse.ac.uk

\begin{abstract}
The different ways of handling a conflict have traditionally been considered as behavioural characteristics of the individual. In this paper, we endeavour to analyse the different ways of handling a conflict in relation to the culture of the organisations. We analyse the culture with three dimensions: high versus low identification of the members with the objectives of the organisation; high versus low power distance; closed versus open system. Combining these three dimensions we elaborate different patterns of organisational culture favouring different forms of conflict behaviour. Departing from these suppositions we accomplished an intervention in a sanitary organisation with the general hypothesis that the culture of this organisation responded to the definition of Pattern 4: high power distance + closed system + low identification. This cultural pattern had to strengthen the behaviour of passive competition, based on resistance and control of the programme which the Basque Public Health Service wanted to impose.
\end{abstract}

\section{Keywords}

Conflict, culture, closed-system, open-system, identification, power. 


\section{INTRODUCTION}

Nowadays it is acknowledged that conflicts, in themselves, are neither constructive or destructive. They are simply the expression of an unresolved problem that causes tension between the individuals or groups making up an organisation. However conflicts, when appropriately managed, can add substantial value to organisations. Conflict can be the medium by which problems are recognised and overcome (Simosi, 1997). Therefore the important thing is not to avoid the appearance of conflicts, but to learn how to manage them. Furthermore an underlying, non-explicit conflict is more difficult to handle than a conflict that is openly expressed.

In organisational settings, employees who discuss conflict disclose information, challenge assumptions, dig into issues, and, as a consequence, are able to make more informed decisions. This is what Tjosvold (1991) calls 'positive conflict'. Through this type of conflict, managers and employees can take a longterm view, anticipate and exploit changes, and keep in touch with each other as well as with customers, suppliers, specialists, and investors. The management of conflict can be indeed a fundamental competitive advantage for the organisation since it helps organisations to develop new strategies.

Organisations certainly need such advantages. We live in a world that it is in constant change. in order to cope with such a reality and 'survive', our organisations have to adapt to that situation by changing themselves. Companies and individuals and groups within those companies confront ongoing change and uncertainty. They need to be poised to appreciate emerging opportunities, circumvent pitfalls and threats, and realise competitive advantages. And one of the key issues in handling this type of change is successful management of conflict situation within the organisational setting.

\section{ORGANISATIONS AND A TAXONOMY FOR MANAGING CONFLICT}

Contemporary organisations are networks of independent units, having 'groups' as their building blocks. For that reason, relations among the individual components of the organisation are complex; they are characterised by a mixture of co-operation and competition. People compete against each other for power and scarce resources but, in the meanwhile, they rely on each other in order to accomplish their tasks, while emotional ties are formed among them (Masterbroek, 1987). Owing to the necessary interdependency among organisational units, effective organising cannot take place, unless such interdependencies are managed effectively and a balance is reached between co-operation and mutual dependency on the one hand, and rivalry and autonomy on the other hand. 
Within such an environment, social interactions among organisational members are complex, since they reflect a fundamental incompatibility (Boulding, 1963): the simultaneous need of competition and co-operation. Consequently as organisations develop and increase size and diversity, different problems emerge. The members of the organisation are likely to have different perspectives about these problems and the conflicts experienced can range from the competition over different resources and struggles for power, to failure to coordinate efforts to achieve a concrete goal or in the way the personal relationships are handled.

Even when the members of the organisation have common interests and agree basically about the goals to be achieved the conflict may arise among them. In any kind of conflict situation people's attempts to manage it will reflect the set of shared assumptions and practices that are in use in a particular organisational context (Garcia and Simosi, 1995).

Moreover, human behaviour in any organisation is of a complex nature, being the product of the interaction between the organisational role expectations and the different personalities of individuals working there (DeCosta, 1991). In every organisation, the formal and informal procedures in terms of rules, norms, beliefs etc., which govern the daily activities if its members and serve to achieve organisational goals, will shape the ways the conflict situations will be handled (Argyris and Schon, 1978). Thus, people working in a specific organisation adhere to the conflict norms in use in that organisation.

Traditionally, the ways of handling conflict (Blake and Mouton, 1964) have been considered mainly as individual behavioural characteristics based, on the one hand, the empirical base of the classical distinction between groups centred on task processes and groups centred on socio-emotional relations (Bales, 1950) and ,on the other hand, the conceptualisation of conflict management based on the following two aspects: (1)Interest in results that favour one's own self; (2) Interest in results that favour the other party.

Combining these two aspects, Thomas and Kilman defined 5 ways of handling conflict: Competition, Collaboration, Compromise, Avoidance, Accommodation. We explore these briefly below:

- Competition: when, in situations of conflict, the individual tends to maintain his or her interests at the cost of others.

- Collaboration: when, in situations of conflict, the individual tends to find halfway solutions that satisfy both parties. Thus, when he or she tries to ensure that "everyone is happy".

- Compromise: when, in situations of conflict, the individual tends to find halfway solutions that satisfy neither him /herself nor the others. Thus, when s/he tries to faily distribute the costs of the conflict among the members of the group.

- Accommodation: when, in situations of conflict, the individual tends to give in to the other person's interests. 
- Avoidance: when in situations of conflict the individual has a greater tendency to try and resolve the problems him or herself.

Recently, authors like Van de Vliert and Euwema(1994) have questioned this manner of approaching the management of a conflict. The reason is apparent: in both the original conceptualisation of Blake and Mouton's and in their subsequent taxonomy of ways of conflict, management the focus is solely on the intentions or inclinations of the each of the subjects. There are a great many factors which play an important role between the intentions of a person and his or her actual behaviour, i.e., the rules of managing the conflict imposed by the group and the role adopted by each individual in the group, as well as factors internal to the person, i.e. the level of self-esteem of the person and earlier training in the handling of a conflict etc. Thus, the behaviour of the individual in situations of conflict doesn't necessarily coincide with his/her previously espoused intentions. This is why Van de Vliert and Euwema prefer to use descriptive aspects closer to behaviour in the handling of a conflict. These aspects are Agreeable/Disagreeable and Active/Passive (Van de Vliert and Euwema, 1994).The operational definitions of these two aspects are based on Bales' Symlog:

- Agreeable: shows agreement; reduces tensions; shows solidarity.

- Disagreeable: shows disagreement; shows tension; shows antagonism.

- Active: offers information; offers suggestions; offers opinions; offers guidance.

- Passive: asks for information; asks for suggestions; asks for an opinion; asks for guidance.

Figure 1 Refined characterisation of modes of conflict behaviour in terms of agreeableness and activeness (Van de Vliert and Euwema, 1994).

\begin{tabular}{|c|c|c|c|}
\hline \multicolumn{3}{|c|}{ COOPERATION } \\
\hline \multicolumn{2}{|c|}{ Passive } & \multicolumn{2}{c|}{ Active } \\
\hline \multicolumn{2}{|c|}{ Nonconfrontation } & \multicolumn{2}{c|}{ Negotiation } \\
\hline Disagreeable & Agreeable & Disagreeable & Agreeable \\
\hline Avoidance & Accommodation & Compromise & Collaboration \\
\hline
\end{tabular}

\begin{tabular}{|c|c|c|c|}
\hline \multicolumn{3}{|c|}{ COMPETITION } \\
\hline \multicolumn{2}{|c|}{ Passive } & \multicolumn{2}{c|}{ Active } \\
\hline \multicolumn{2}{|c|}{ Indirect Fighting } & \multicolumn{2}{c|}{ Direct Fighting } \\
\hline Agreeable & Disagreeable & Agreeable & Disagreeable \\
\hline $\begin{array}{c}\text { Process } \\
\text { Controlling }\end{array}$ & Resistance & Issue Fighting & $\begin{array}{c}\text { Outcome } \\
\text { Fighting }\end{array}$ \\
\hline
\end{tabular}


In this taxonomy we must pay attention to three aspects. Cooperative behaviour includes a certain amount of competition: disagreeable behaviour, such as avoidance and compromise, mean that cooperation is being sought, but without eliminating competitive feelings. On the other hand, accommodation and the solving of problems demonstrate more harmonious cooperation. In competition, disagreeable behaviour, such as control of the group process and issue fighting mean that there is less competition than when disagreeable resistance and outcome fighting behaviour are used.

This taxonomy of ways of managing a conflict demands that, when observing this behaviour, consideration be given both to the actions and to the emotional state of the members of the group. There is also a fourth aspect we need to take into account when considering or analysing conflict in organisational settings. This is the context in which that conflict is trying to be managed: the "cultural context" of the organisation.

We do not regard organisational members as being acted upon by the work environment. Instead, we just advocate that their own motivations and cognitions necessarily operate in a social context (Condor and Brown, 1988). To understand this, we have to view communicative interactions among organisational members as a process of enactment of a shared reality (Morgan, 1986). Organisational life consists of a set of assumptions and expectations towards one another, which determine both formal and informal interactions within the organisational setting (Garcia and Humphreys, 1995). Such a shared reality serves as a means of communication among organisational members. Issues such as organisational structure, job descriptions, policies and operating procedures have interpretative function, since they enable organisational members to shape the organisational reality (Morgan, 1986).

\section{CONFLICT MANAGEMENT WITHIN A CULTURAL FRAMEWORK}

Various ways of management of conflict have traditionally been considered just as the behavioural characteristics of the individual (McDougall, 1920). In this work, we endeavour to analyse the different ways of managing conflict in relation to the wider social context where those conflict situations occur, i.e., in relation to the culture of the organisation (Turner, 1987).

Usually, the members of an organisation belong to several and overlapping cultural frames of reference where organisational reality, as such, is only one part of the daily life. As members of a particular social group, class, region or country we bring into the organisation different cultural influences. From this point of view it could be difficult to explore the influence the culture of the organisation has in the different ways the individuals manage organisation-related conflicts. However if we take into account that the organisation is in itself the "nexus" where those 
broader, societal, "feeder" cultural frames of reference come together, the consequence is that although many external cultural influences permeate the organisation's boundary, each organisation has a particular combination of cultural influences which mixes and interacts within a given organisational boundary.

It is true that any kind of organisation, just like any living being, needs to define its social identity in order to establish the limits of where it belongs and to mark the differences between "them" and "us". The members of the organisation define their identity by specifying the characteristics that distinguish them from other groups. They tend to define themselves as "unique" or "different" or "special" in contrast to other groups or organisations . The "identity" of the organisation and the group becomes strengthened by established rituals of admittance and behavioural rules which those wishing to belong to the group must follow. This does not mean that we consider the group or the organisation as a closed system: quite the contrary, we are aware that the boundaries of any organisation tend to be permeable and in a sense arbitrary, thus quite open. This type of boundary allows us to consider, nevertheless, some degree of "uniqueness", because boundaries are about space demarcation even though the space within a boundary is versatile, multiple and mobile. At the same time they have the unique quality of preserving differences and identities whereas allowing interchange or "contamination".

However, when an organisation or group is too deeply concerned with defining and strengthening its identity, due to uncertainty, change or perceived threats etc., it has the tendency to fall back on itself and develop self-defence mechanisms which eventually convert the group into a closed social system (Ayestarán, 1996). These behaviour-regulating mechanisms of the group members are as follows:

- The group becomes the only group reference for its members. The membranes of the group become impermeable to outside information.

- Interpersonal interaction is reduced in order to control the risk of conflict within the group.

- Conflictive behaviour in relation to outside groups is reinforced and cognitive processes of favouritism within the group is bolstered. The figure of the prototype who ends up assuming the role of charismatic leader is strengthened.

- The "black sheep" mechanism is set in motion, so that members seen as the cause of the group's problems are pushed aside or expelled.

- Functional opposition between personal identity and social identity is accentuated, thus calling for behaviour which is coherent with the social identity of the members and condemns the strengthening of personal selfesteem.

All of these mechanisms have one sole purpose: to control the individual interests of the members of the group and to achieve a unity of action revolving around the objectives of that action. The result is that the group does not allow its members to demonstrate direct confrontational behaviour in conflict management; instead, it 
favours compliant behaviour, avoidance and indirect confrontation. This can become a progressively dysfunctional defence as the group isolates itself from understanding the process which triggered the defence mechanism and therefore distancing itself from a constructive participation individually and/or collectively in it.

This is the context in which individual/group conflict arises, the purpose of which is to strengthen autonomy of the individual with regard to the group, thus bringing about a cultural and structural change in the latter.

\section{SOCIAL INTERACTION AND THE DEVELOPMENT OF A NEW CULTURAL FRAMEWORK WITHIN THE ORGANISATION}

This type of individual/group conflict can be overcome in two ways:

a) By changing the prototype of the group, thereby implying a purely structural change - the access to power of a new subgroup arranged around a new prototype. The group continues with the same identity and hierarchical structure that it had before the conflict. The group leader changes but the culture of the group remains the same as before. Changes of this kind often occur in political parties, religious institutions and professional groups.

b) By changing the group culture. For this to happen, the group encourages interaction and direct confrontation between its members, the negotiation of cognitive perspectives and personal interests in relation to the group objectives, the solving of problems and the integration of individual objectives into the new group objectives. Here, the change is both structural and cultural, and involves:

- Questioning the earlier definition of the group. Questioning allows the group to work out a new definition for itself that is more compatible with the demands of the cultural, economic and social environment.

- A change in the power structure, that is to say, a closer relationship between the leader and the members making up the group.

- Greater participation by all members in defining the objectives of the group.

- Restructuring the activities of the group, placing greater importance on its objective interdependence as far as tasks are concerned.

- The group no longer being the only reference group for its members. They can take references from other groups.

- An increase in internal group conflict. The conflicts are interpersonal and affect the values of the group as well as its objectives and the rules governing its internal interactions.

- A reduction in intergroup conflict and the creation of new conditions for intergroup collaboration. 
In a sense, this type of socio-cognitive conflict and the change that follows it, involves two tendencies: one that aims at maintaining existing frameworks and agreement, and the other that aims at changing them by imparting an original form to things and ideas. This will serve the double purpose of offering a familiar and secure background to connect the new ideas and events while, at the same time, offering the necessary foreground where individuals, through their reflective awareness, will be able to make choices, manipulate and even innovate their conditions, paving the ground for a new intergroup collaboration.

\section{DIALECTIC IDENTIFICATION/INTERACTION}

Any group of human beings must maintain its identity, while, however, responding to the demands of the environment and allowing for the personal growth of its members. When the pole of identity is stressed, the group favours nonconfrontational behaviour and indirect confrontation between its members. Group cohesion then takes the shape of identification with group objectives and rules. This kind of cohesion is not good when it comes to managing differences between individuals and tends to unify their thoughts and actions (groupthink), thereby implying a loss of creativity and initiative, as well as the group's capacity to respond to the demands of the environment. The chances increase that poor decisions will be made and the group becomes a closed social system.

The change of cultural frameworks within the group implies greater interaction between its members, and transforms a potential conflict between the individuals and the group into a conflict between its members. Individual interests take on greater relevance and competition puts cooperation and the very existence of the group into danger.

\section{A SYSTEMIC PERSPECTIVE OF THE GROUP}

Until now, the systems theory has produced little relevant research in the area of groups and organisations, and it can be said that no new knowledge concerning intra- and inter-group dynamics has been presented. However, the systemic perspective permits better integration of our knowledge to the area of groups. We believe that there are two important concepts regarding the overcoming of individual/group opposition and the relation of intragroup dynamics to intergroup dynamics. 
The open or closed group systems refer to the way in which groups handle their internal differences, deviations from their rules and intragroup conflicts.

- The group as a closed system involves: circular stable internal relations; stable balance; the maintenance of identity; self-referential relations; a reduction in internal fluctuations; control of the environment from inside the system; the absence of reciprocal relations with other systems; the hierarchical organisation of relations between systems. It also implies a reduction in constructive interpersonal interaction and the avoidance of conflict.

- The group as an open system involves: dynamic and unstable internal relations; unstable balance; structuring at a higher level of balance; reciprocal relations between systems; an increase in fluctuations; influence of the environment on the system; inter-systemic interaction; a lack of hierarchicallyorganized inter-systemic relations.

Applying these concepts to social systems helps us to learn about the role played by both intra- and inter-group conflict in the construction of social reality and its evolution towards more complex forms of operation.

Isomorphism between the different subsystems means that the dynamics of development and conflict are similar for the individual, group and inter-group subsystem. This means that any changes introduced to one of the subsystems will affect the dynamics of the other subsystems.

\section{THE INFLUENCE OF CULTURE ON MODES OF CONFLICT BEHAVIOUR}

Depending on the psycho-social model of the group, we can define group culture on the basis of three variables:

- High/low power distance: High power distance reduces social interaction inside the organisation. Low power distance increases social interaction.

- High/low group identification: High group identification reduces individual autonomy. Low group identification increases individual autonomy.

- Closed system/open system: Closed systems reduce intragroup conflict and increase intergroup conflict. Open systems increase intragroup conflict.

In the following diagrams, we demonstrate the influence of group culture on decision taking. 
- Pattern 1: low power distance + closed system + high identification: Conflict avoiding behaviour and the maintenance of group harmony.

- Pattern 2: low power distance + closed system + low identification: Conflict avoiding behaviour and the reduction of interpersonal interaction.

- Pattern 3: high power distance + closed system + high identification: Accommodating conflict behaviour.

- Pattern 4: high power distance + closed system + low identification: Process controlling and resisting conflict behaviour.

- Pattern 5: high power distance + open system + high identification: Compromising conflict behaviour.

- Pattern 6: high power distance + open system + low identification: Issue fighting conflict behaviour.

- Pattern 7: low power distance + open system + high identification: problem solving conflict behaviour.

- Pattern 8: low power distance + open system + low identification: outcome fighting conflict behaviour.

The highest standard of conflict behaviour occurs when group culture is defined in terms of low power distance, the group functions as an open system, and there is high identification with the group by its members.

\section{APPLICATION OF THE MODEL TO PSYCHOSOCIAL INTERVENTION.}

Below we present an intervention carried out by Cristina Martínez-Taboada in the Basque Public Health Service to illustrate the utility of the model presented for the intervention.

\subsection{Request}

The request for intervention on behalf of the Nursing Department of the Basque Public Health Service came as a result of the persistent failure to implement a mother/child care programme supposedly to be operated by the midwives belonging to the outpatients network. Paradoxically, the programme sought to introduce the very bases for development, evaluation and systematic functioning that the midwives had themselves long been clamouring for. The request for intervention involved:

- Talking to the midwives group to see if resistance to the new programme could be diminished

- Generating a group dynamic to analyse the difficulties in the implementation of the programme.

- Finding possible solutions to these difficulties. 


\subsection{Hypotheses}

We started from the general hypothesis that the culture of this group responded to the definition of Pattern 4: HIGH POWER DISTANCE + CLOSED SYSTEM + LOW IDENTIFICATION. This cultural pattern had to strengthen the behaviour of passive competition, based on resistance and control of the programme which the Basque Public Health Service wanted to impose.

- An analysis of the situation confirmed our initial hypothesis: The culture of the group, in a closed system, resists change.

- This resistance manifests itself in the failure to implement the new programme.

- The programme is not seen as meeting the group's demands, but on the contrary as an imposition from above.

\subsection{Objectives of the intervention.}

Based on the suppositions we have just set out, the intervention was planned to achieve the following objectives:

- To set up a group dynamic that would permit the group to become aware of its internal manner of functioning and of the repercussion of this manner of functioning on its relations with other groups in the Institution. The objective was to succeed in making the group receptive to information from other groups and, in this way, to relate to these groups' positions. This is what we understand by "converting a closed system into an open system".

- To underline the differences of opinion and of existing positions within the same group, without its members being able to express these differences. This manner of functioning indicates to what point the group of midwives was reproducing the hierarchical culture of the Health Institution.

- To analyse, as objectively as possible, the positive and negative aspects of the programme imposed by the health authorities, trying to assess the programme itself, while putting the influence of the conflict with the authorities to one side. The purpose was to achieve greater identification of the midwives with the objectives of the mother/child care programme and bring them closer to the objectives of the Health Institution.

\subsection{The intervention process.}

The intervention began by acknowledging the professional and personal interests shared by the group, and which formed the basis of its motivation, looking at the goals, expectations and working conditions of each group member. Our attention soon focused on the common denominator of the sociostructural conditions: the hierarchic set-up of the Institution, in going over the relation between the group 
and superiors - and looking at the many ways in which different individuals had contributed towards impeding the new programme- the hitherto stable defensive balance began to give way.

This process enabled the group members to question their own attitude towards the programme, and to recognise the psychosocial factors which gave its implementation a somewhat menacing aura.

Of these factors, the following were found to be fundamental to the intervention:

- the programme was seen as a means by which superiors sought to further their own interests;

- therefore any hindrance of the programme thwarted such interests;

- professional diffiaulties were al ways blamed on superiors;

- therefore the midwives' group itself was never to blame.

Rising awareness of these psychosocial factors gave group members an increased ability to take a personal stand with regard to both the job and the programme, and the degree of intragroup conflict became apparent. Conflict between the different levels of hierarchy assumed a lesser role. The participants became more able to view the programme as a group task, to examine it dispassionately and subsequently take a decision. This process was greatly helped by the Institution's decision to consult the group of midwives. The group could negotiate certain aspects such as the materials to be used.

\subsection{Changes obtained}

- The midwives contributed to the implementation of the programme.

- Commonly accepted methods of evaluating the systemisation of the programme were established.

- The programme was implemented in Gipuzkoa and subsequently, using the same intervention design, in the rest of the Basque Autonomous Community.

\section{CONCLUSION}

The objective of the intervention was to change the behaviour of the midwives as regards their conflict with the health authorities. It was specifically an attempt to pass from a style of resistance and control of the process to an open confrontation and to a behaviour based on compromise and negotiation.

This change of behaviour was achieved thanks to the cultural change which had previously been achieved with the group. The intervention was directed at achieving this cultural change. The cultural change implied: 
- Transformation of the internal dynamic of the group of midwives, making it more open to outside information and offering it the possibility to demonstrate the discrepancies between its members.

- Reduction in the degree of hierarchical organisation of the Institution by creating channels of dialogue and negotiation between the group of midwives and the Management of the Basque Public Health Service.

- Separation of the assessment of the task from the negative emotions associated with conflict with the authorities, thereby facilitating greater identification with the objectives of the programme and of the Health Institution.

\section{REFERENCES}

Argyris, C. and Schon, D. (1978) Organisational Learning: A Theory of Action Perspective. Addison-Wesley, Reading, Mass.

Ayestarán, S. (1996) El grupo como construcción social. Barcelona, Ediciones Plural.

Bales, R. F. (1950) Interaction process analysis: a method for the study of small groups. Addison Wesley, Cambridge, Mass

Blake, R. R. and Mouton, J. S. (1964) The Managerial Grid. Gulf, Houston.

Boulding, K. (1963) Conflict and Defence. Harper and Row, New York.

Condor, S. and Brown, R. (1988) Psychological processes, in The Social Psychology of Intergroup Conflict (eds. W. Stroebe, A. W. Kruglanski et al.). Springer-Verlag, New York.

DeCosta, J. (1993). Conflict Management and the Learning Organisation: A Case Study. PhD Thesis. The Fielding Institute, California, USA.

Garcia, L. and Humphreys, P. (1995) Major Organisational change and its Impact in culture, in People, Technology and Productivity: New Visions and Strategies for the Next Century. National Productivity Centre, Turkey.

Garcia, L. and Simosi, M. (1995) Managing Undergoing Cultural Change in Organisations .Unpublished presentation for the IX European Congress in Social Psychology. Athens, Greece.

Mastenbroek, W.F. (1987) Conflict Management and Organisation Development. Holland: Wiley.

McDougall, W. (1920) The Group Mind. Putnam, New York

Morgan, G. (1986) Images of Organisation. Sage, London.

Simosi, M. (1997) The Processing of Conflict in Organisational Groups: A Case

Study in a Greek Industrial Company. PhD Thesis. Social Psychology Department, The London School of Economics. London, U.K.

Tjosvold, D. (1991) The Conflict-Positive Organisation. Addison-Wesley, London. Turner, J. C. (1987) Rediscovering the Social Group: A SelfCategorisation Theory, Basil Blackwell, Oxford. 
Van de Vliert, E. and Euwema, M. C. (1994) Agreeableness and Activeness as Components of Conflict Behaviours. Journal of Personality and Social Psychology, 66, 674-687.

\section{BIOGRAPHIES}

Sabino Ayestarán, Cristina Martínez-Taboada and Juanjo Arrospide form a team of professors in Social Psychology in the University of the Basque Country in Spain. Their research interests are related to the Psychology of groups and the Psychology of the organisations. In their theoretical research, they have developed a psychosocial model of group that tries to integrate Self-Categorisation Theory and Social Interaction Theory with support from Systems Theory. In this context, they have devoted many projects to the analysis of the conflicts, in the interpersonal level as well as in the organisational and political level.

Lucia Garcia is a researcher in the Social Psychology Department at the London School of Economics (LSE), working on organisational change and its impact on the culture of organisations. She also teaches Organisational Psychology and Qualitative Research Methods for the Methodology Institute at LSE. She has participated in a number of research projects in Latin America and Eastern Europe regarding organisational transformation in those countries. Currently she is a research officer on the WHO Healthy Cities Evaluation Project. 